\title{
Comparative Assessment of Diagnostic Performances of Two Commercial Rapid Diagnostic Test Kits for Detection of Plasmodium spp. in Ugandan Patients with Malaria
}

\author{
Young Yil Bahk', Seo Hye Park², Woojoo Lee ${ }^{3}$, Kyoung Jin", Seong Kyu Ahn², Byoung-Kuk Na, Tong-Soo Kim³ \\ ${ }^{1}$ Department of Biotechnology, College of Biomedical and Health Science, Konkuk University, Chungju 27478, Korea; ${ }^{2}$ Department of Tropical \\ Medicine, Inha University College of Medicine, Incheon 22212, Korea, ${ }^{3}$ Department of Statistics, Inha University College of Natural Sciences, \\ Incheon 22212, Korea; ${ }^{4}$ Korea Health Evaluation Institute, Sahmyook University, Seoul 01795, Korea; ${ }^{5}$ Department of Parasitology and Tropical \\ Medicine, and Institute of Health Sciences, Gyeongsang National University College of Medicine, Jinju 52727, Korea
}

\begin{abstract}
Prompt diagnosis of malaria cases with rapid diagnostic tests (RDTs) has been widely adopted as an effective malaria diagnostic tool in many malaria endemic countries, primarily due to their easy operation, fast result output, and straightforward interpretation. However, there has been controversy about the diagnostic accuracy of RDTs. This study was conducted to evaluate the diagnostic performances of the 2 commercially available malaria RDT kits, RapiGEN Malaria Ag Pf/Pv (pLDH/pLDH) and Asan EasyTest ${ }^{\mathrm{TM}}$ Malaria Ag Pf/Pv (HRP-2/pLDH) for their abilities to detect Plasmodium species in blood samples collected from Ugandan patients with malaria. To evaluate the diagnostic performances of these 2 RDT kits, 229 blood samples were tested for malaria infection by microscopic examination and a species-specific nested polymerase chain reaction. The detection sensitivities for $P$. falciparum of Malaria Ag Pf/Pv (pLDH/pLDH) and Asan EasyTest $^{\mathrm{TM}}$ Malaria Ag Pf/Pv (HRP-2/pLDH) were $87.83 \%$ and $89.57 \%$, respectively. The specificities of the 2 RDTs were $100 \%$ for $P$. falciparum and mixed $P$. falciparum/P. vivax infections. These results suggest that the 2 RDT kits showed reasonable levels of diagnostic performances for detection of the malaria parasites from Ugandan patients. However, neither kit could effectively detect $P$. falciparum infections with low parasitaemia ( $<500$ parasites $/ \mu l)$.
\end{abstract}

Key words: Malaria, rapid diagnostic test, diagnostic performance, Uganda

\section{INTRODUCTION}

Malaria is the most prevalent parasitic disease in the world, with an estimated 3.2 billion people in danger of being infected and half of the world's population at risk of contracting malaria [1]. In 2016, although the incidence of malaria has decreased in recent years, there were an estimated 216 million cases of malaria, an increase of about 5 million cases over 2015 and deaths reached 445,000, a similar number to the previous year [2]. Despite significant control efforts, morbidity and mortality induced by malaria remain high in many developing countries, especially in areas characterized by tropical and subtropical ecosystems. Among the 5 Plasmodium species of human malaria parasites, $P$. falciparum is the most dangerous and

- Received 3 June 2018, revised 15 August 2018, accepted 5 September 2018.

*Corresponding author (tongsookim@inha.ac.kr)

(c) 2018, Korean Society for Parasitology and Tropical Medicine

This is an Open Access article distributed under the terms of the Creative Commons Attribution Non-Commercial License (http://creativecommons.org/licenses/by-nc/4.0) which permits unrestricted non-commercial use, distribution, and reproduction in any

medium, provided the original work is properly cited. can cause severe clinical manifestations and even death [3]. P. vivax accounts for almost half of the malaria cases worldwide and is no longer considered as a mild infection [4]. Accurate malaria diagnosis is practically the only tool of effecting rational therapy. It is important to have sensitive and specific malaria diagnostic tools to prevent injudicious use of anti-malaria drugs and overtreatment. Microscopic examination of blood smears has been used as a gold standard for malaria diagnosis in many malaria endemic areas, despite its limitations on infrastructural and technical requirements that are not always available in resource-limited settings. Inconsistency due to intense inter-observer variability, particularly for samples with low parasitaemia or mixed Plasmodium species, has been regarded as a major shortcoming of microscopic examination [5-7]. These limitations have fostered the development of non-microscopic alternatives for the diagnosis of malaria, especially in field diagnosis. Although molecular methods based on polymerase chain reaction (PCR) allow for highly accurate diagnosis for malaria, these methods cannot be easily applied for point of care applications. Therefore, rapid diagnostic tests (RDTs) are 
increasing their role as diagnostic aid in microscopic diagnosis of malaria in many endemic areas owing to their considerable advantages in field application [8,9]. The global availability and scale of use of RDTs has gradually increased over recent decades [10]. RDTs are immunochromatographic tests capable of detecting the antigens of one or more Plasmodium species. Although the use of malaria RDTs has improved global malaria control due to their ease of use and fast result interpretation, the accurate and adequate reporting of malaria cases for monitoring malaria trends remains a challenge in resource-limited nations [11]. In addition, commercially available RDTs do not present marked sensitivity over microscopic tools, given that their overall sensitivity is diminished when the parasitaemia level is low [12]. Moreover, their usefulness is limited owing to their inability to quantify parasitaemia. This study aimed to evaluate the quality of 2 commercially available malaria RDTs for their abilities to detect Plasmodium species in malaria cases from Uganda. Although their use in many public and private clinics in Korea and several countries has grown in recent years, their diagnostic performances in field settings have not been fully established, especially for clinical isolates from a variety of malaria endemic countries.

In this study, we assessed the diagnostic accuracy of these RDTs by comparing the sensitivity, specificity, and positive and negative predictive values of these RDTs with those of microscopic examinations and species-specific nested PCR in symptomatic Ugandan patients with malaria.

\section{MATERIALS AND METHODS}

\section{Ethics statement, study design, and population}

All participants and patients were informed of this study and signed informed consent was obtained according to ethical standards. Prior to blood collection, applicable information was provided to patients, including information about the procedures themselves and the potential risks and benefits of this study. Young children under 8 years old, pregnant women, and patients with signs of severe and complicated malaria infections, as defined by WHO [13], were excluded from this study. This study was implemented in accordance with the principles of the Helsinki Declaration. Sampling was performed between April and September 2016 in primary health centers in the malaria endemic Kiyuni Parish of Kyankwanzi District, Uganda. Participants were sampled as previously described [14].

\section{Microscopic examinations and nested PCR}

The blood samples obtained from patients with malaria $(\mathrm{n}=229)$ were screened by microscopy and species-specific nested PCR for species identification and determination of parasite density before performing the RDT examination. For microscopy examination, thick and thin capillary blood smears were prepared as previously described [14]. Smears were stained with a $4 \%$ Giemsa solution ( $\mathrm{pH} 7.2$ ) for $20 \mathrm{~min}$. All smears were independently prepared by 3 trained Ugandan research technicians following the standard protocols $[15,16]$. The microscopic analysis was also confirmed by the Department of Tropical Medicine, Inha University College of Medicine to minimize diagnostic errors. Parasite density was calculated by counting against 299-500 white blood cells and esti-

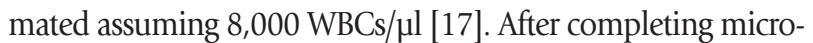
scopic examination, all blood sample was used for PCR analysis. Genomic DNA was isolated from blood samples using QIAamp DNA blood kit (Qiagen, Hilden, Germany) according to the manufacturer's instructions. All samples from patients showing a positive microscopy result were further tested by species-specific nested PCR targeting Plasmodium $18 \mathrm{~S}$ ribosomal RNA gene (18S rRNA), as described previously $[5,18]$.

\section{RDTs}

Samples were used to detect malaria parasitaemia by blood smear and by nested PCR analysis. The RDTs used in this study were the RapiGEN Malaria Ag Pf/Pv (HRPII/pLDH) (Cat. No. H006087, RapiGEN INC, Gunpo, Korea) and the Asan Easy Test $^{\mathrm{TM}}$ Malaria Ag Pf/Pv Strip (Cat. No. H2015, Asan Pharmaceutical Co. Ltd., Hwaseong, Korea). The used RDT kits were having the same Lot. No. for the accurate comparison. The Asan EasyTest ${ }^{\mathrm{TM}}$ Malaria Ag Pf/Pv (HRP-2/pLDH) detects the histidine-rich protein-2 (HRP-2) antigen specific for $P$. falciparum HRP-2 and Plasmodium lactate dehydrogenase (pLDH). The RapiGEN Malaria Ag Pf/Pv (pLDH/pLDH) detects various isomers of $\mathrm{LDH}$ antigens for $P$. falciparum and $P$. vivax. Each RDT test was performed following the manufacturer's instructions. Test line intensities, which were visible as cherry-red to purple-colored lines, were scored according to each RDT as belonging to one of 4 categories: none (no line visible), weak (paler than the control line [+]), medium (equal to the control line [++]) and strong (stronger than the control line [+++]). All results were followed strictly adhering to the manufacturers' instructions. There were no invalid test results. The evaluation process maintained an unbiased methodology. The status of 
the samples was unknown to the persons involved in performing the pre-analytical and analytical procedures. To evaluate the specificity and sensitivity of each RDT on the basis of microscopic examination and nested PCR results, a study was performed on a sample panel consisting of 229 clinical samples collected from Ugandan patients with malaria.

\section{Data analysis}

The results from 2 different RDT kits were evaluated by microscopic examination as known as gold standard method of blood samples. $P$-values less than 0.05 were considered statistically significant. Sensitivity and specificity were calculated to assess the diagnostic performance of 2 RDTs compared to microscopy examinations and nested PCR. The sensitivity was defined as the percentage of positive results among samples confirmed to be positive by microscopic examinations. The specificity was the percentage of negative results among samples whose thick blood smears were negative.

\section{RESULTS}

Field tests were conducted in 6 villages of the Kiyuni Parish in Kyankwanzi District, Uganda in 2016. A total of 229 blood donor samples were obtained from the study participants. Of the 229 samples (the mean age was $21.13 \pm 11.19$ years old; male= 93; female=136), 114 were negatives for both $P$. falciparum and $P$. vivax by microscopic examination and nested PCR analyses (Table 1). Expert microscopic examination de-

Table 1. Comparison of diagnostic performance of two RDT kits with microscopic examination and nested PCR

\begin{tabular}{|c|c|c|c|c|c|c|c|c|c|}
\hline & \multicolumn{2}{|c|}{ Microscopy } & \multicolumn{3}{|c|}{ PCR } & \multicolumn{2}{|c|}{ RDT $1 *$} & \multicolumn{2}{|c|}{ RDT $2^{*}$} \\
\hline & - & $P f$ & - & $P f$ & Mixed $^{\star \star}$ & - & $P f$ & - & $P f$ \\
\hline Negative & 114 & & 114 & & & 114 & & 114 & \\
\hline Positive & & 103 & & 103 & 12 & 14 & 101 & 12 & 103 \\
\hline Subtotal & 114 & 103 & 114 & 103 & 12 & 128 & 101 & 126 & 103 \\
\hline Total & 229 & & 229 & & & 229 & & 229 & \\
\hline
\end{tabular}

${ }^{*}$ RDT1: Rapigen, Pf/Pv (pLDH/pLDH). RDT2: Asan EasyTest, Pf/Pv (HRP-2/pLDH).

${ }^{* *}$ Mixed: Pf/Pv.

Table 2. Diagnostic performance of two RDT kits by parasitemia

\begin{tabular}{|c|c|c|c|c|c|c|c|c|c|c|}
\hline \multirow{3}{*}{ Parasites $/ \mu l$} & \multicolumn{5}{|c|}{ RDT Pf/Pv (pLDH/pLDH) (RapiGEN) } & \multicolumn{5}{|c|}{ RDT Pf/Pv (HRP-2/pLDH) (Asan EasyTest) } \\
\hline & Weak & Medium & Strong & $-*$ & Total & Weak & Medium & Strong & $-*$ & Total \\
\hline & \multicolumn{10}{|c|}{ P. falciparum } \\
\hline$>100,000$ & - & - & 6 & 1 & 7 & - & - & 5 & 2 & 7 \\
\hline $10,000-100,000$ & 1 & 3 & 20 & - & 24 & 1 & 1 & 22 & - & 24 \\
\hline $5,000-10,000$ & - & 1 & 8 & 1 & 10 & - & 2 & 7 & 1 & 10 \\
\hline $3,000-5,000$ & - & 2 & 12 & 1 & 15 & - & 3 & 11 & 1 & 15 \\
\hline $1,000-3,000$ & 6 & 8 & 5 & 2 & 21 & 3 & 8 & 8 & 2 & 21 \\
\hline $500-1,000$ & 2 & 5 & 3 & 3 & 13 & 2 & 5 & 3 & 3 & 13 \\
\hline $100-500$ & 4 & 3 & 1 & 5 & 13 & 5 & 4 & 2 & 2 & 13 \\
\hline$<100$ & - & - & - & - & - & - & - & - & - & - \\
\hline \multirow[t]{2}{*}{ Total } & 13 & 22 & 55 & 13 & 103 & 11 & 23 & 58 & 11 & 103 \\
\hline & \multicolumn{10}{|c|}{ Mixed } \\
\hline$>100,000$ & - & - & - & - & - & - & - & - & - & - \\
\hline $10,000-100,000$ & - & 3 & 3 & - & 6 & - & 4 & 2 & - & 6 \\
\hline $5,000-10,000$ & 2 & - & 1 & - & 3 & 1 & 1 & 1 & - & 3 \\
\hline $3,000-5,000$ & - & - & - & - & - & - & - & - & - & - \\
\hline $1,000-3,000$ & - & - & 1 & - & 1 & - & - & 1 & - & 1 \\
\hline $100-1,000$ & - & 1 & - & 1 & 2 & - & - & 1 & 1 & 2 \\
\hline$<100$ & - & - & - & - & - & - & - & - & - & - \\
\hline Total & 2 & 4 & 4 & 1 & 12 & - & - & - & - & 12 \\
\hline
\end{tabular}

*negative. 
tected 115 samples as being infected with $P$. falciparum $(\mathrm{n}=103)$ or showing mixed infections of $P$. falciparum and $P$. $\operatorname{vivax}(\mathrm{n}=12)$. Microscopic examination and nested PCR analyses for 115 patients who tested positive for malaria suggested that $P$. falciparum infection was highly prevalent: $P$. falciparum infections $(103 / 115,89.57 \%)$ and mixed infections of $P$. falciparum and $P$. vivax $(11 / 115,10.43 \%)$. The mean age of the patients with malaria was $17.6 \pm 10.69$ years (Range: $2-61$ years); females accounted for $58.83 \%$ of the total number of patients with malaria. Patients were distributed across all age groups, but the most highly represented patient group was the 10-19 year-old group (43/115 participants [37.39\%]), followed by patients from the 0-9 (29/115 [25.22\%]) and 20-29 (22/115, [19.13\%]) year-old groups. The geometric mean parasitaemia level was 19,948.56 parasites/ul; the maximum was 376,800 parasites $/ \mu \mathrm{l}$, and the minimum was 320 parasites $/ \mu l$. The most prevalent parasitaemia group was the $10,000-100,000(30 / 115)$ parasites/ $\mu$ group, followed by the 1,000-3,000 (22/115) para-

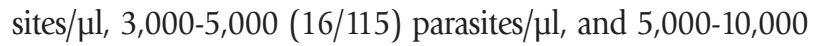
(13/115) parasites/ $\mu$ groups. Blood samples from 114 participants were used to evaluate the specificity of the test kits. The mean age of the negative participants was 24.61 years (standard deviation: 10.08; range: 6-65 year of age) and comprised of 69/114 females (60.53\%) (Table 2). Study participants belonged to all age groups, but the most prevalent participant group was that of the 20-29 year-old group (44/114 participants [38.60\%]), followed by the 10-19 year-old group (33/114 [28.95\%]), and the 30-39 year-old group (25/114 [21.93\%]). The P. falciparum specificities for RapiGEN and Asan RDT brands were $100 \%$. Overall, the P. falciparum sensitivities for RapiGEN and Asan RDT brands were 88\% (101/115 patients, (specificity with 95\% CI, Likelihood ratio+ [LR+] ; 80\%, Likelihood ratio- [LR-]; 93\%) and 89.57\% (103/115, Likelihood ratio+ [LR+]; 82\%, Likelihood ratio- [LR-]; 94\%) among the samples diagnosed as having $P$. falciparum and mixed malaria infections, respectively (Tables 1,2 ). The positive and negative predictive values for RapiGEN RDT kits were 100\% (LR+: 96\%, LR-: 100\%) and 89\% (82\%, 94\%), respectively and those for Asan RDT kits were 100\% (96\%, 100\%) and $90 \%$ (84\%, 95\%), respectively, whereas the negative likelihood ratios for each kit were $12 \%(7 \%, 20 \%)$ and $10 \%$ (6\%, $18 \%$ ) and accuracies for each kit were $94 \%$ and $95 \%$, respectively. As definition, positive likelihood ratio is infinitive because the P. falciparum specificity was $100 \%$.

\section{DISCUSSION}

This study was conducted to measure the overall diagnostic performances of 2 RDT kits for Plasmodium infection. Patient blood samples were confirmed as either positive (115 patients) or negative (114 participants), in order to determine the appropriate test for field use Uganda. To be useful and provide an efficient diagnostic method, RDTs must exhibit greater than 95\% sensitivity according to WHO recommendation [19]. Overall, the P. falciparum sensitivities for RapiGEN and Asan RDT brands were $87.83 \%$ (101/115 patients) and $89.57 \%$ (103/115) among the samples diagnosed as having $P$. falciparum and mixed malaria infection, respectively (Tables 1, 2). P. falciparum sensitivities for RapiGEN and Asan RDT brands were found to be $61.54 \%$ ( $8 / 13$ patients) and $84.62 \%$ (11/13) for parasite density $>500$ parasites/ $\mu$ l and $94.44 \%(93 / 90)$ and $90.00 \%$ (81/90) for $<500$ parasites $/ \mu l$, respectively. Similarly, the sensitivities for the mixed infection samples were $91.67 \%$ (11/12) for the 2 test kits. In addition, the sensitivity for the Asan brand kit was found to decline for P. falciparum at a parasite density $<100,000$ parasites $/ \mu l$. Intensities of test line with 10,000-1,000,000 parasites/ $\mu$ l densities were found to be reduced in the $P$. falciparum samples for the RapidGEN brand and in the Pf mixed samples for the Asan brand, respectively (Table 2). It is practically acceptable that comparative assessment is difficult because [1] reference standards are dissimilar; [2] clinical epidemiological characteristics of study populations; [3] trials do not employ common guidelines; [4] the timing of the study could influence the prevalence of malaria in the population; and [5] products of different lots may differ in quality $[20,21]$. According to previously published RDT study reports, HRP-2 assays commonly provide P. falciparum sensitivity of $>90 \%$ in clinical cases [22-24]. Study of HRP-2based RDTs can be influenced by several factors, including antigenic variability of the target protein, antigen persistence in the bloodstream following elimination of parasites, and parasite density below the RDT threshold of detection [25-27]. Reassuringly, a recent trial evaluating 8 independent HRP-2based RDTs in Western Kenya reported sensitivities that were comparable to microscopic examination of 90-95\% [28]. In the case of $\mathrm{pDDH}$ assays, although product lots and variable field stability of the test kits could not be ruled out, the test results varied among studies. Sensitivity for $P$. falciparum is excellent ( $>95 \%$ ) in some studies and poorer (more than $80 \%$ ) in other studies [12,29]. In general, it is acceptable that overall 
RDT specificity is commonly observed to be $85 \%$ [12]. In this study, although there is a limitation that it has been done on a relatively small scale and in a limited area experiment, these RDT kits we have used could be a good alternative diagnostic tool, given the cost, technical, and local circumstances of PCR and microscopic examinations. However, it is also necessary to obtain the results of a study that can be generalized by examining and comparing different regions over a long period of time. In addition, compared to the other brand RDT kits that are now using for the diagnosis of Plasmodium species, there are same limitations of poor detection of mixed infection, of all species in Plasmodium and of the antigen in specimen continued after the treatment.

In conclusion, the present study reported the diagnostic performances of 2 commercially available malaria RDTs, RapiGEN Malaria Ag Pf/Pv (pLDH/pLDH) and Asan EasyTest ${ }^{\mathrm{TM}}$ MalariaAg Pf/Pv (HRP-2/pLDH), for blood samples collected from Ugandan patients with malaria. The overall diagnostic accuracy of the RDTs against $P$. falciparum was comparatively similar to that of RDTs recommended by WHO for a parasite density $<500$. The sensitivity for samples with mixed infection was $91.67 \%$ for both RDTs. Therefore, the 2 RDT kits evaluated in this study appear to be relatively reliable diagnostic tools to detect $P$. falciparum infections in Uganda. However, further measurement is needed to fully evaluate the diagnostic performances of these 2 RDTs, including a more extensive examination of samples from various global regions and populations.

\section{ACKNOWLEDGMENT}

This work was supported by the Inha University Research Fund.

\section{CONFLICT OF INTEREST}

The authors declare that they have no competing interests.

\section{REFERENCES}

1. Snow RW, Guerra CA, Noor AM, Myint HY, Hay SI. The global distribution of clinical episodes of Plasmodium falciparum malaria. Nature 2005; 434: 214-217.

2. World Health Organization. World Malaria Report, 2017. Geneva, Switzerland. World Health Organization. 2017.

3. Singh B, Daneshvar C. Human infections and detection of Plasmodium falciparum knowlesi. Clin Microbiol Rev 2013; 26: 165-
184.

4. Rogerson SJ, Carter R. Severe vivax malaria: newly recognised or rediscovered. PLoS Med 2008; 5: e136.

5. Kang JM, Cho PY, Moe M, Lee J, Jun H, Lee HW, Ahn SK, Kim TI, Pak JH, Myint MK, Lin K, Kim TS, Na BK. Comparison of the diagnostic performance of microscopic examination with nested polymerase chain reaction for optimum malaria diagnosis in Upper Myanmar. Malar J 2017; 16: 119.

6. Ohrt C, Sutamihardja MA, Tang D, Kain KC. Impact of microscopy error on estimates of protective efficacy in malaria-prevention trials. J Infect Dis 2002; 186: 540-546.

7. Moody A. Rapid diagnostic tests for malaria parasites. Clin Microbiol Rev 2002; 15: 66-78.

8. D'Acremont $\mathrm{V}$, Lengeler $\mathrm{C}$, Mshinda $\mathrm{H}$, Mtasiwa D, Tanner $\mathrm{M}$, Genton B. Time to move from presumptive malaria treatment to laboratory-confirmed diagnosis and treatment in African children with fever. PLoS Med 2009; 6: e252.

9. Doudou MH, Mahamadou A, Ouba I, Lazoumar R, Boubacar B, Arzika I, Zamanka H, Ibrahim ML, Labbo R, Maiguizo S, Girond F, Guillebaud J, Maazou A, Fandeur T. A refined estimate of the malaria burden in Niger. Malaria J 2012; 11: 89.

10. WHO. Information Note on Recommended Selection Criteria for Procurement of Malaria Rapid Diagnostic Tests (RDTs). Geneva, Switzerland. World Health Organization. 2016.

11. Afrane YA, Zhou G, Githeko AK, Yan G. Utility of health facilitybased malaria data for malaria surveillance. PLoS One 2013; 8: e54305.

12. Wongsrichanalai C, Barcus MJ, Muth S, Sutamihardja A, Wernsdorfer WH. A review of malaria diagnostic tools: microscopy and rapid diagnostic test (RDT). Am J Trop Med Hyg 2007; 77: 119-127.

13. World Health Organization. Monitoring Antimalarial Drug Resistance. Report of WHO Consultation 2001.

14. Chong CK, Cho PY, Na BK, Ahn SK, Kim JS, Lee JS, Lee SK, Han ET, Kim HY, Park YK, Cha SH, Kim TS. Evaluation of the accuracy of the EasyTest ${ }^{\mathrm{TM}}$ Malaria Pf/Pan $\mathrm{Ag}$, a rapid diagnostic test, in Uganda. Korean J Parasitol 2014; 52: 501-505.

15. Warhurst DC, Williams JE. Laboratory diagnosis of malaria. J Clin Pathol 1996; 49: 533-538.

16. World Health Organization. Management of Uncomplicated Malaria and the Use of Antimalarial Drugs for the Protection of Travellers. Report of an Informal Consultation Geneva, 18-21 September 1995. Geneva, Switzerland. World Health Organization. 1997

17. Brown AE, Kain KC, Pipithkul J, Webster HK. Demonstration by the polymerase chain reaction of mixed Plasmodium falciparum and $P$. vivax infections undetected by conventional microscopy. Trans R Soc Trop Med Hyg 1992; 86: 609-612.

18. Snounou G, Singh B. Nested PCR analysis of Plasmodium parasites. Methods Mol Med 2002; 72: 189-203.

19. World health Organization. Malaria Diagnosis New Perspectives. Report of a Joint WHO/USAID Informed Consultation, 25-27 October 1999. Geneva, Switzerland. World Health Orga- 
nization. 2000, pp 57.

20. Murray CK, Gasser RA Jr, Magill AJ, Miller RS. Update on rapid diagnostic testing for malaria. Clin Microbiol Rev 2008; 21: 97110.

21. Gillet P, van Dijk DP, Bottieau E, Cnops L, van Esbroeck M, Jacobs J. Test characteristics of the SD FK80 Plasmodium falciparum/ Plasmodium vivax malaria rapid diagnostic test in a non-endemic setting. Malaria J 2009; 8: 262.

22. Kilian AH, Mughusu EB, Kabagambe G, von Sonnenburg F. Comparison of two rapid, HRP2-based diagnostic tests for Plasmodium falciparum. Trans R Soc Trop Med Hyg 1997; 91: 666667.

23. Killian AH, Kanagambe G, Byamukama W, Langi P, Weis P, von Sonnenburg F. Application of the ParaSight-F dipstick test for malaria diagnosis in a district control program. Acta Trop 1999; 72: 281-293.

24. Guthmann JP, Ruiz A, Priotto G, Kiguli J, Bonte L, Legros D. Validity, realiability and ease of use in the field of five rapid tests for the diagnosis of Plasmodium falciparum malaria in Uganda. Trans R Soc Trop Med Hyg 2002; 96: 254-257.
25. Lee N, Gatton ML, Pelecanos A, Bubb M, Gonzalez I, Bell D, Cheng Q, McCarthy JS. Identification of optimal epitopes for Plasmodium falciparum rapid diagnostic tests that target histidinerich proteins 2 and 3. J Clin Microbiol 2012; 50: 1397-1405.

26. Houzé S, Boly MD, Le Bras J, Deloron P, Faucher JF. PfHRP2 and PfLDH antigen detection for monitoring the efficacy of artemisinin-based combination therapy (ACT) in the treatment of uncomplicated falciparum malaria. Malaria J 2009; 8: 211.

27. Cheng Q, Gatton ML, Barnwell J, Chiodini P, McCarthy J, Bell D, Cunningham J. Plasmodium falciparum parasites lacking histidine-rich protein 2 and 3: a review and recommendations for accurate reporting. Malar J 2014; 13: 283.

28. Wanja EW, Kuya N, Moranga C, Hickman M, Johnson JD, Moseti C, Anova L, Ogutu B, Ohrt C. Field evaluation of diagnostic performance of malaria rapid diagnostic tests in western Kenya. Malaria J 2016; 15: 456.

29. Singh N, Valecha N, Nagpal ACMishra SS, Varma HS, Subbarao SK. The hospital- and field-based performance of the OptiMAL test, for malaria diagnosis and treatment monitoring in central India. Ann Trop Med Parasitol 2003; 97: 5-13. 